\title{
Comments to Drs. Gastaldon, Raschi, Kane, Barbui, and Schoretsanitis
}

\author{
Teodora Doherty ${ }^{\mathrm{a}}$ Ella J. Daly ${ }^{\mathrm{b}}$ Janice Miller $^{\mathrm{a}}$ Vanina Popova $^{\mathrm{c}}$ \\ M. Soledad Cepeda ${ }^{d}$ Wayne C. Drevets ${ }^{e}$ Carla M. Canuso $^{e}$ \\ ${ }^{a}$ Global Medical Safety, Janssen Research \& Development, LLC, Titusville, NJ, USA; ${ }^{b}$ Neuroscience Medical \\ Affairs, Janssen Scientific Affairs, LLC, Titusville, NJ, USA; ' Neuroscience Clinical Development, Janssen Research

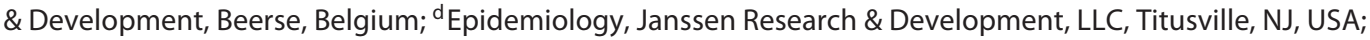 \\ eNeuroscience Clinical Development, Janssen Research \& Development, LLC, Titusville, NJ, USA
}

We acknowledge the work of Gastaldon et al. [1], who used the US Food and Drug Administration (FDA) Adverse Event Reporting System (FAERS) to identify potential safety signals related to esketamine. Janssen recognizes esketamine's unique safety profile and is committed to continuous assessment of postmarketing safety data through comprehensive pharmacovigilance surveillance from multiple sources, including FAERS. Moreover, as required by the FDA, Janssen follows an ongoing Risk Evaluation and Mitigation Strategy (REMS) [2] to mitigate potential risks of serious adverse outcomes associated with sedation, dissociation, and misuse/abuse of esketamine.

The authors' interpretation of their findings is overstated given the substantial limitations of FAERS, a postmarketing surveillance program for voluntary reporting of adverse events (AE). FAERS commonly contains duplicate, incomplete, and/or unverified AE reports from which causality cannot be determined [3]. Further, AE rates cannot be determined from FAERS [3] since reporting is voluntary and unsolicited, resulting in underreporting for most drugs [4]. In contrast, the esketamine REMS requires solicited reporting of sedation, dissociation, and all serious $\mathrm{AE}$ at every treatment session, resulting in multiple reports of an $\mathrm{AE}$ per patient.

karger@karger.com
www.karger.com/pps
Karger $\%$
BOPEN Access

(C) 2020 The Author(s)

Published by S. Karger AG, Basel

This is an Open Access article licensed under the Creative Commons Attribution-NonCommercial-4.0 International License (CC BY-NC) (http://www.karger.com/Services/OpenAccessLicense), applicable to the online version of the article only. Usage and distribution for commercial purposes requires written permission.
Gastaldon et al. [1] noted 4 main findings. First, they highlighted an "increasing trend for the number of esketamine reports" over the analysis period. This trend is expected given the low initial esketamine usage following approval, based partly on requirements for treatment center certification to ensure REMS compliance. Second, the authors highlighted that their "analysis detected rare AEs, which were not reported by regulatory trials." Since esketamine is used concomitantly with an oral antidepressant(s) and often in patients with comorbidities, reported AEs cannot necessarily be attributed to esketamine. Third, the authors highlighted "strong and consistent disproportionality estimates for several AEs," especially for dissociation and sedation. As noted above, if these expected AEs occur, they are reported at every treatment session via REMS, driving multiple reports in FAERS. Fourth, the authors highlighted "high reporting ratios of suicidal ideation" and "completed suicide." The FAERS database does not include information about baseline suicidality, illness severity, or comorbidities risk factors for suicidal ideation/behavior. Importantly, patients with treatment-resistant depression attempt suicide at a rate 7 -fold higher than that in patients with treatment-responsive depression [5]. Although the authors conducted a sensitivity analysis using venlafaxine as the

Teodora Doherty, MD

Global Medical Safety, Janssen Research \& Development, LLC

1125 Trenton-Harbourton Rd.

Titusville, NJ 08560 (USA)

tdoherty@its.jnj.com 
control, interpretation of findings from this analysis is limited because esketamine is indicated for patients with treatment-resistant depression, who manifest a substantially greater burden of disease than patients for which venlafaxine is indicated [6]. Moreover, epidemiological data [7] collected postapproval showed that at baseline esketamine-treated patients, versus other patients with treatment-resistant depression, exhibited $\geq 2$-fold higher rates of severe depression, anxiety disorder, and suicidal thoughts. Finally, while the authors raise the potential for misuse/abuse, no such signal has been identified from comprehensive surveillance.

In summary, our comprehensive surveillance has not revealed any new safety signal and confirms that Spravato ${ }^{\circledR}$ product labeling [8] adequately addresses esketamine's risks. Additionally, data are being collected in a prospective long-term safety study (NCT02782104). Janssen remains committed to ongoing esketamine safety monitoring via robust risk management and pharmacovigilance surveillance programs, including REMS, to ensure that up-to-date safety information is available to prescribers and patients.

\section{Acknowledgements}

We acknowledge Sandra Norris, PharmD of the Norris Communications Group LLC, supported by Janssen Research and Development, LLC, for medical writing assistance, and Ellen Baum, PhD (Janssen Global Services, LLC), for additional editorial support.

\section{Conflict of Interest Statement}

All of the authors are employees of Janssen Research \& Development, LLC, or Janssen Scientific Affairs, LLC, and stockholders of Johnson \& Johnson, Inc.

\section{Author Contributions}

T.D., E.J.D., J.M., V.P., M.S.C., W.C.D, and C.M.C. contributed equally to this letter, including writing and approval of the text for submission.

\section{References}

1 Gastaldon C, Raschi E, Kane JM, Barbui C, Schoretsanitis G. Post-marketing safety concerns with esketamine: a disproportionality analysis of spontaneous reports submitted to the FDA adverse event reporting system. Psychother Psychosom. 2020 Aug;1-8.

2 Spravato ${ }^{\circledR}$ REMS (Risk Evaluation and Mitigation Strategy). [cited 2020 Sep 4]. Available from: https://www.spravatorems.com.

3 US Food and Drug Administration. FDA Adverse Event Reporting System (FAERS) public dashboard. [cited 2020 Sep 4]. Available from: https://www.fda.gov/drugs/questionsand-answers-fdas-adverse-event-reportingsystem-faers/fda-adverse-event-reportingsystem-faers-public-dashboard.
4 Toki T, Ono S. Spontaneous reporting on adverse events by consumers in the United States: an analysis of the Food and Drug Administration Adverse Event Reporting System database. Drugs Real World Outcomes. 2018 Jun;5(2):117-28.

5 Feldman RL, Dunner DL, Muller JS, Stone DA. Medicare patient experience with vagus nerve stimulation for treatment-resistant depression. J Med Econ. 2013;16(1):62-74.
6 Effexor $\mathrm{XR}^{\circledR}$ (venlafaxine extended release) prescribing information. [cited 2020 Sep 4]. Available from: http://labeling.pfizer.com/ showlabeling.aspx?id=100.

7 IBM $^{\circledR}$ MarketScan ${ }^{\circledR}$ Commercial Claims \& Encounters (CCAE). Available from: https:// www.ibm.com/downloads/cas/6KNYVVQ2.

8 Spravato $^{\circledR}$ (esketamine) nasal spray prescribing information. ${ }^{(} 2020$ Janssen Pharmaceutical Companies. Available from: http://www. janssenlabels.com/package-insert/productmonograph/prescribing-information/ SPRAVATO-pi.pdf. 